\title{
Modeling of biomass productivity in dense microalgal culture using computational fluid dynamics
}

\author{
P. Fernández-del Olmo1,a, J.M. Fernández-Sevilla1', F.G. Acién'1, A. González-Céspedes², \\ J.C. López-Hernández² and J.J. Magán² \\ ${ }^{1}$ Engineering Department, University of Almeria, Almería, Spain; 2Estación Experimental de Cajamar Las \\ Palmerillas, Fundación Cajamar - Grupo Cooperativo Cajamar, Almería, Spain.
}

\begin{abstract}
Biomass productivity is the variable to optimize in microalgal processes and is strongly related to light availability inside the photobioreactor. The so-called "light regime" in dense microalgal cultures is the result of the movement of microalgal cells inside the irradiance field caused by the stirring of the culture. Proper mixing increases productivity and helps microalgal cells to move frequently between dark and light zones but it is not trivial to ascertain the adequate level of mixing for a particular microalgal strain and culture system. Computational Fluid Dynamics (CFD) allows a precise description of the irradiance field inside a microalgal culture and makes it possible to calculate the movement of a population of single cells as a function of geometry and mixing intensity. Thus, realistic light-time patterns can be obtained and their effects on the photosynthetic response of microalgae can be analysed. In this work, we present the analysis of an actual horizontal tubular photobioreactor whose mixing patterns are obtained by CFD and are coupled with a dynamic model of photosynthesis for Nannochloropsis gaditana to determine biomass productivity. This data is compared to the experimental data of biomass productivity obtained in the same photobioreactor to show that the model developed agrees with the experimental results within a $20 \%$ error margin.
\end{abstract}

Keywords: computational fluid dynamics, microalgae, photobioreactor, photosynthesis model

\section{INTRODUCTION}

Microalgae-based processes have received an increasing attention due to their potential to produce biomass at a high output rate using $\mathrm{CO}_{2}$ and solar light. Microalgae contain a large number of high value molecules such as carotenoids as lutein or asthaxantin and long-chain polyunsaturated fatty acids (LC-PUFAs) such as EPA or DHA. In recent years the interest in microalgae processes has shifted to lower value commodities such as biofertilizers and biofuels (Chisti, 2007) but the engineering efforts done in that direction have highlighted the need of more efficient production systems and in particular the need of more research in photobioreactor (PBR) development, which is the core of these processes.

One of the difficulties in PBR design is that the high-density cultures needed to assimilate the high irradiances supplied by the sun cause self-shadowing inside the culture and thus the coexistence of dark zones with very illuminated zones. In this situation, a poor mixing causes decrease in photosynthetic yield as the fraction of microalgae that stay in the illuminated zones becomes saturated or even photo inhibited while in the dark zones cells can be photo-limited so that photosynthesis is slowed to a halt. Good mixing promotes regular movement of microalgae from light to dark zones increasing photosynthetic yield as cell are exposed to an adequate average irradiance. In this sense, Computational Fluid Dynamics (CFD) is a unique tool as it allows evaluating the performance of a PBR before it has been built (Bitog et al., 2011). In this work we show how a CFD description of a PBR coupled with a dynamic model of photosynthesis (Camacho-Rubio et al., 2003) with growth

${ }^{a}$ E-mail: pfd793@ual.es 
parameters obtained in laboratory can be used to predict the productivity of a pilot-plant scale PBR.

\section{MATERIALS AND METHODS}

\section{Photobioreactor}

The horizontal tubular photobioreactor used was made with a loop of $90 \mathrm{~m}$ length built with PMMA pipe (internal diameter of $0.0455 \mathrm{~m}$ ) and PVC in curves. The PBR was operated in semi-continuous mode with microalga Nannochloropsis gaditana. Biomass concentration was maintained close to $\mathrm{Cb}=2060 \mathrm{~g} \mathrm{~m}^{-3}$ by periodical dilutions. The liquid velocity in the tubular loop was $1.7 \mathrm{~m} \mathrm{~s}^{-1}$. The PBR was located in the Experimental Station "Las Palmerillas" of Cajamar (latitude $36^{\circ} 48^{\prime} \mathrm{N}$, longitude $2^{\circ} 43^{\prime}$ O, altitude $151 \mathrm{~m}$ ) (El Ejido, Almería, Spain). A view of the PBR is shown in Figure 1.

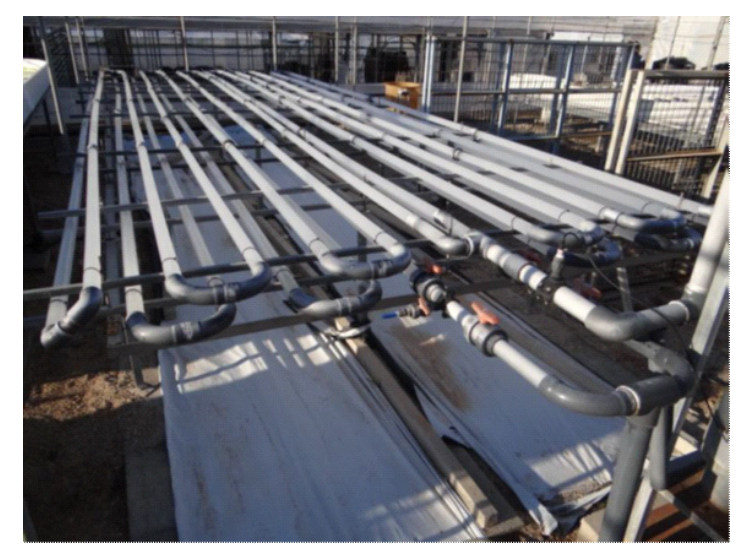

Figure 1. View of the experimental tubular photobioreactor.

\section{CFD model}

The photobioreactor was investigated using commercial CFD software. A hexahedral grid was used for a suitable discretization of the computational domain. The discretization in the circumferential direction was uniform, but the radial distribution was chosen in order to increase the number of elementary volumes close to walls. The mesh was previously subjected to a sensitivity analysis to obtain mesh independent result and reduce the discretization errors and the computational time. For this purpose six meshes with different element density were built and evaluated. The tubular PBR was meshed after determining the meshing parameters that gave better results in terms of computational time and accuracy. The resulting grid was composed of $13.510^{6}$ cells (Figure 2).

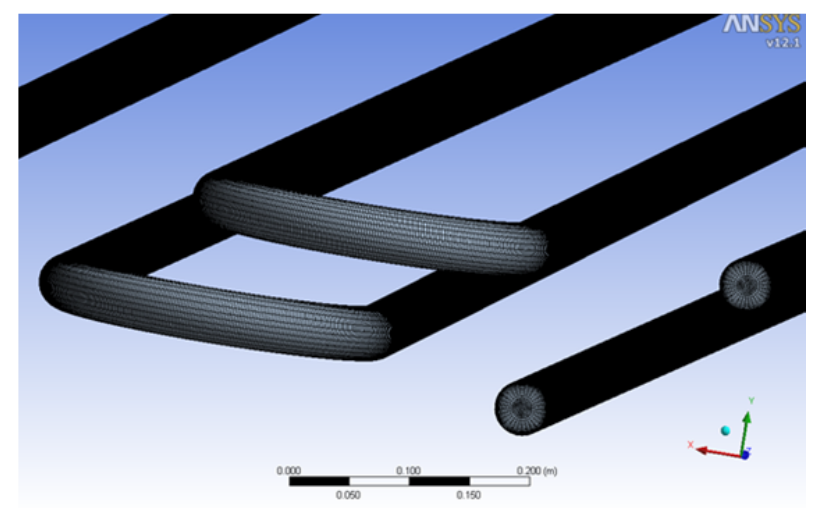

Figure 2. Hexahedral type mesh built for the CFD model. 
The transport equations were solved using the software package Ansys Fluent 12.1. The numerical model is based on the finite-volume-method. The flow solution procedure is the Semi-Implicit Method for Pressure-Linked Equation SIMPLE routine, developed by Patankar (1980). The momentum equations are discretized using both first and second order upwind scheme options, and second-order up-wind for other transport equations. Computational domain is discretized using Ansys Meshing 12.1. The convergence criterion consisted of monitoring mass flow rate on inlet and outlet and variation of velocity profiles with iteration, reduction of several orders of magnitude in the residual errors. To perform realistic simulation of turbulence, various turbulence models have been tested. The most accurate precision was achieved using RSM with linear pressure-strain submodel and enhanced wall treatments as the near-wall treatment.

The incident radiation was simulated by the Discrete Ordinates (DO) model included in the CFD software. This model permits the control of the angular discretization of the space and is the only radiation model included in the Ansys Fluent software that permits the distribution of the incident radiation in different wavelength ranges. Besides, this model offers more accuracy in the simulation of the radiation interaction with semi-transparent media (reactor walls), although it requires a more precise definition of the optical properties of the materials integrating the computational domain as well as a much higher computational cost and calculation time.

In order to validate the CFD model, the results were compared with data obtained by Veenman (2004) and Walpot et al. (2007), who investigated the turbulent pipe flow following a numerical approach based on direct numerical simulations (DNS) and experimental technique 3D particle tracking velocimetry (Figure 3).
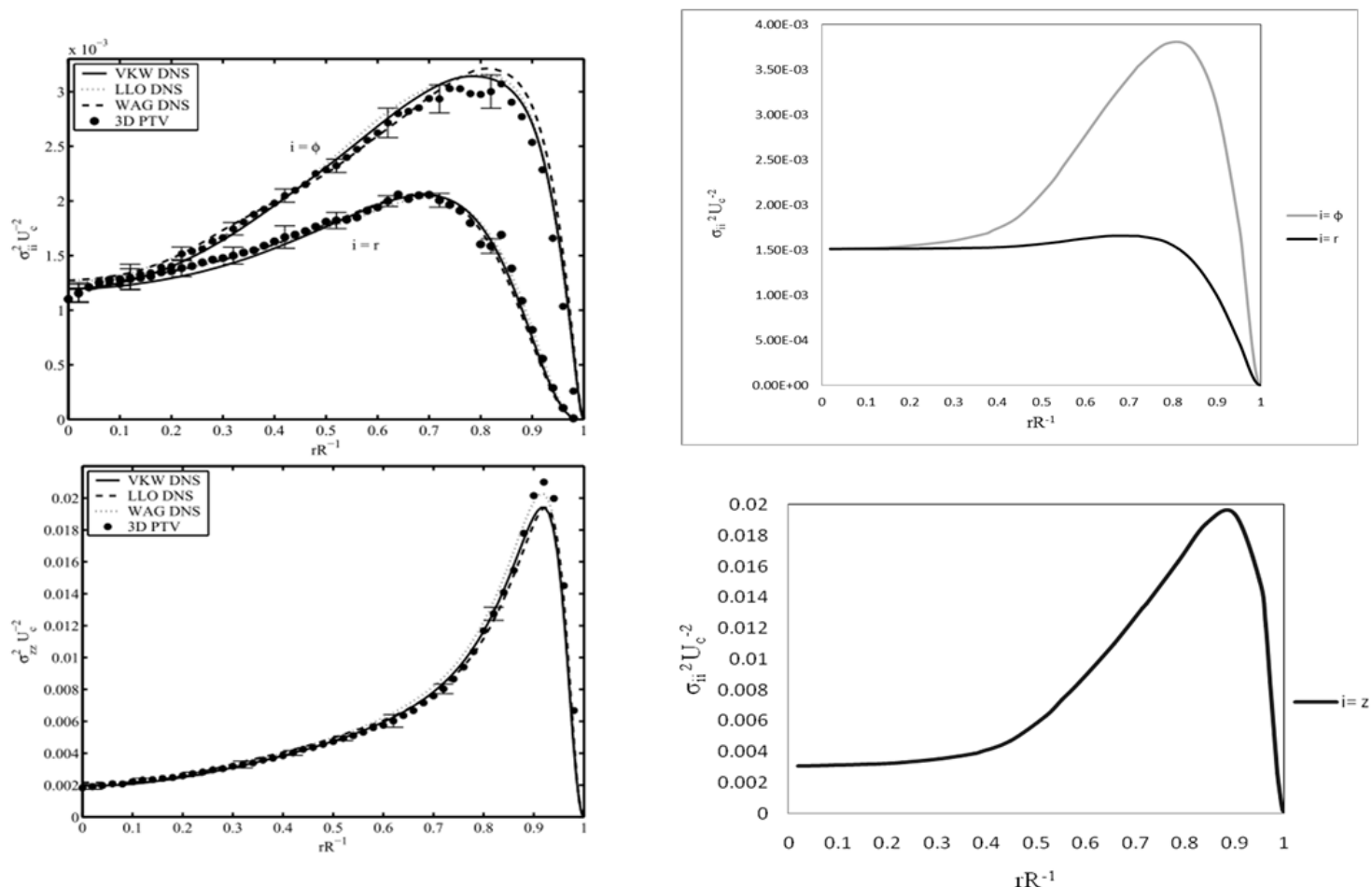

Figure 3. Hexahedral Profiles of fluctuating velocity components (radial, $r$, and tangential, $\Phi$ (top) and axial, z (down)) obtained in the CFD simulations using Reynolds Stress Model (right) and achieved by Veenman (2004) and Walpot et al. (2007) (left).

For the validation of the radiation simulations, experimental data were measured in horizontal tubular PBR for microalgae cultivation. A submergible spherical PAR radiation microsensor was used (model US-SQS/L, Walz, Germany) to determine the radiation inside 
the PMMA pipes.

The cell trajectories inside the PBR were calculated in a Lagrangian frame of reference applying Discrete Random Walk Model (DRW) provided by the software. This is a stochastic tracking model that accounts for the eddy lifetime and that can predict the dispersion of particles due to turbulence in the fluid phase. All model parameters were adapted to the properties of algal cells. The irradiance patterns received by the cells in their trajectory along the reactor were investigated by integrating a Lagrangian approach with the radiation model.

\section{Dynamic photosynthesis growth model}

The irradiance pattern $\mathrm{I}(\mathrm{t})$ obtained by coupling the cell trajectories with the irradiance distribution was used to calculate the photosynthetic response of the microalgae Nannochloropsis gaditana with the dynamic Photosynthesis model proposed by Camacho Rubio et al. (2003). This model consists of the two following equations.

$$
\begin{gathered}
\frac{d x *}{d t}=\frac{\beta}{\alpha} \cdot \mathrm{I}(\mathrm{t}) \cdot\left(1-x^{*}\right)-\beta \frac{x *}{k+x *} \\
P O_{2}=P O_{2 \max } \cdot \frac{x *}{k+x *}
\end{gathered}
$$

Where the growth model parameters $\alpha$ (light saturation constant), $\beta$ (a characteristic frequency), $\kappa$ (a growth saturation constant) and $\mathrm{PO}_{2 \max }$ (the maximum photosynthetic response) can be obtained with an experimental procedure as described by Brindley et al. (2011). This dynamic model was integrated with the light pattern obtained by CFD, $\mathrm{I}(\mathrm{t})$, as input variable using Matlab to obtain the photosynthetic response $\left(\mathrm{PO}_{2}\right)$.

\section{RESULTS AND DISCUSSION}

\section{Determination of trajectories by CFD}

The "light regime" that describes the variation of irradiance with time, $\mathrm{I}(\mathrm{t})$, to which microalgal cells are exposed, is a consequence of the movement of the fluid elements inside the light gradient within the PBR. The first step to obtain I $(\mathrm{t})$ is thus to describe movement of individual microalgae in the PBR. This has been done by simulating by CFD the trajectories of a population of individual microalgal cells moving in the PBR described above at $1.7 \mathrm{~m} \mathrm{~s}^{-1}$ axial speed. These cells were represented by $5 \mu \mathrm{m}$ spheres with a $998.2 \mathrm{~kg} \mathrm{~m}^{-3}$ density. The results obtained are displayed in Figure 4. This figure shows the trajectories of a population of 50 individual cells moving in the PBR loop. The data obtained from the simulation allows calculating (not shown for conciseness) that the radial movement, the one that induces the changes from light to dark zones, is relatively slow and a significant movement from the center to the surface only occurs once every two seconds (frequency of $0.5 \mathrm{~Hz}$ ) which shows that there is ample room for improvement as microalgal cells grow close to their maximum efficiency only at frequencies of $5 \mathrm{~Hz}$ and above (Brindley et al., 2011). The simulation also shows that the elbows at the tube bends induce significant radial mixing that is beneficial with regard to the light regime but at the cost of an increase in head loss.

\section{Irradiance distribution and light regime}

The presence of suspended biomass in the culture causes an intense attenuation of light due to the high content of photosynthetic pigment microalgae cells contain. This is what causes the light gradient I $(\mathrm{x}, \mathrm{r}, \varphi)$ shown in Figure 5. Since the PBR loop is a cylinder placed horizontally and illuminated from above by the sun, the most intensely illuminated zone is the upper part of the cylinder. This intense gradient is caused by a biomass concentration of $2060 \mathrm{~g} \mathrm{~m}^{-3}$. As can be seen in the Figure 5, the light gradients are very intense, demonstrating the need for a correct mixing. 


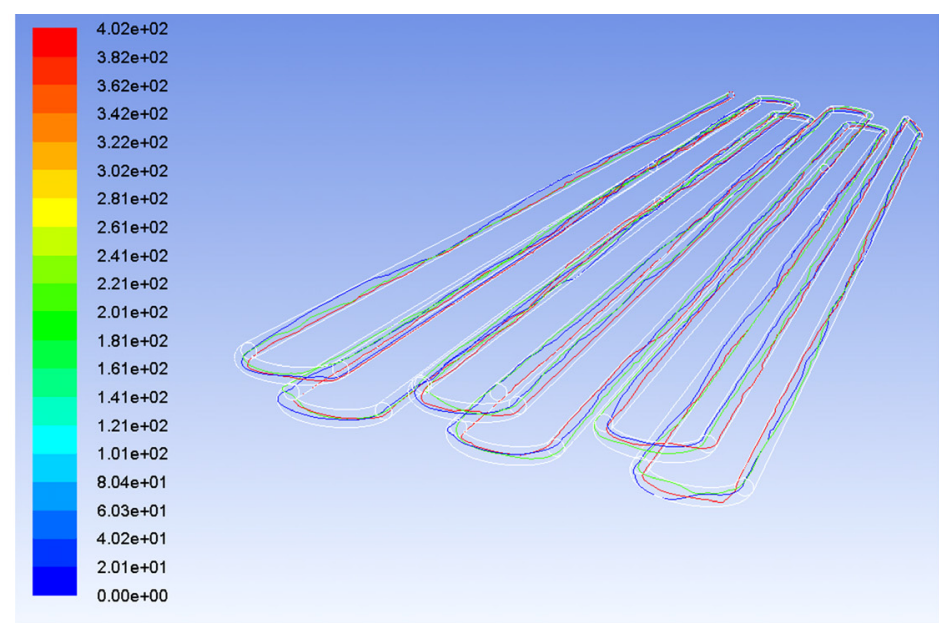

Particle Traces Colored by Particle ID

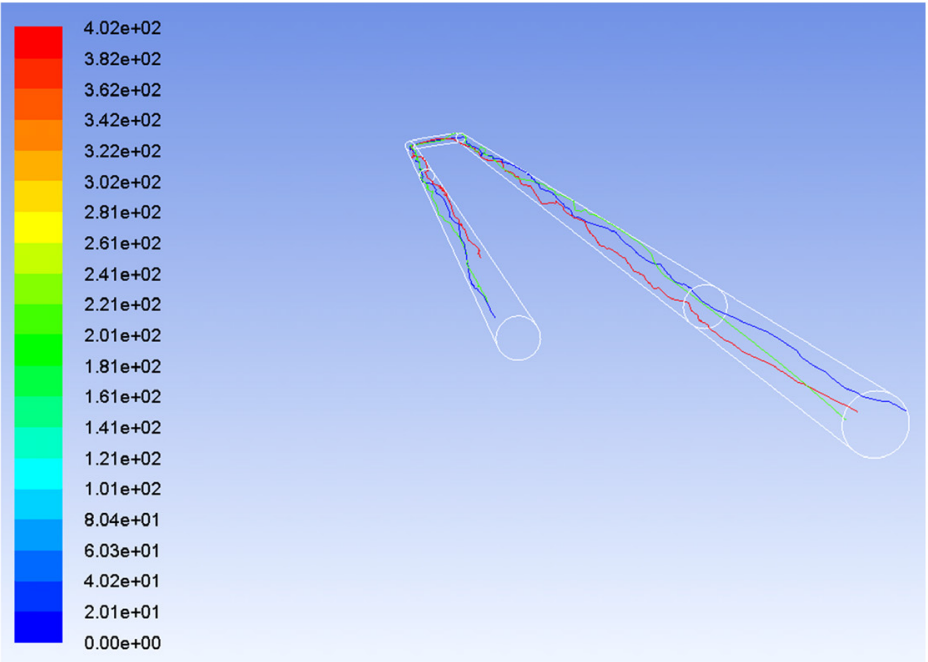

Particle Traces Colored by Particle ID

Figure 4. Simulated trajectories in the $0.0455 \mathrm{~m}$ diameter tubular PBR with $1.7 \mathrm{~m} \mathrm{~s}^{-1}$ axial velocity.

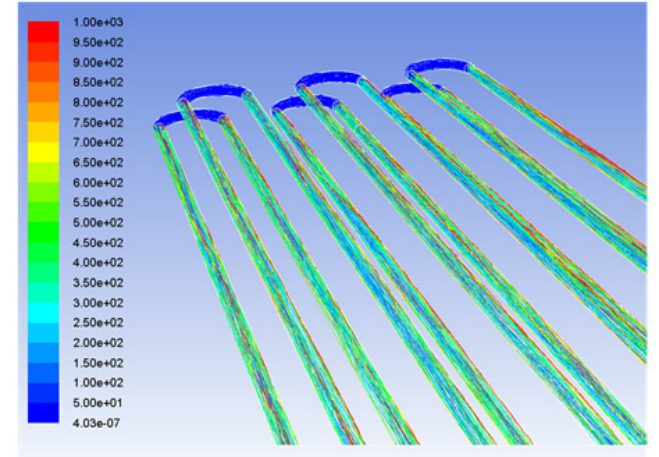

Particle Traces Colored by Incident Radation (w/m2)

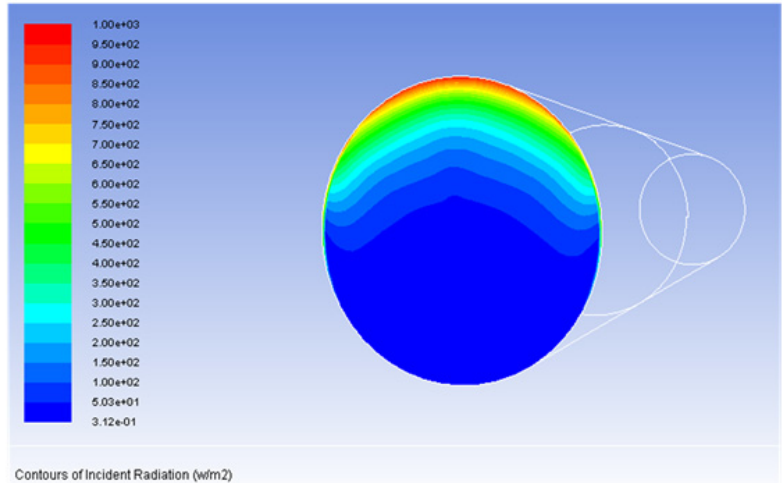

Contours of Incident Radiation wim?

Figure 5. Irradiance distribution inside the photobioreactor calculated for an external irradiance of $\mathrm{I}_{0}=953.5 \mathrm{~W} \mathrm{~m}^{-2}$ and a biomass concentration $\mathrm{Cb}=2060 \mathrm{~g} \mathrm{~m}^{-3}$. 
It is noteworthy that the maximum irradiance level in the cylinder goes above $1200 \mathrm{~W}$ $\mathrm{m}^{-2}$ which is in point of fact higher than the solar irradiance ( $\left.953.5 \mathrm{~W} \mathrm{~m}^{-2}\right)$. This is caused by the concentration of the solar beams impinging on the PMMA surface that is correctly reproduced by the CFD program and would have gone disregarded if the calculation had been done by the methods usually proposed in the literature. This highlights the superiority of CFD applied to the design of PBRs.

\section{Simulation results and validation}

The coupling of the information of the two previous steps results in the exposition pattern I $(\mathrm{t})$ received by the simulated particles. Figure 6 shows $60 \mathrm{~s}$ of the light regime of a single particle sampled at $100 \mathrm{~Hz}$ as an example. This continuously varying irradiance on a microalgal cell elicits a photosynthetic response that can be calculated with the use of a dynamic photosynthesis model such as the proposed by Camacho-Rubio et al. (2003) with the growth parameters displayed in Table 1.

Table 1. Growth parameters of the model proposed by Camacho-Rubio et al. (2003) for Nannochloropsis gaditana at two temperatures.

\begin{tabular}{|c|c|c|c|}
\hline \multirow{2}{*}{ Growth parameter } & \multicolumn{2}{|c|}{ Temperature } & \multirow{2}{*}{ Unit } \\
\hline & $25^{\circ} \mathrm{C}$ & $20^{\circ} \mathrm{C}$ & \\
\hline $\mathrm{PO}_{2 \max }$ & 4.46 & 5.58 & $\mu \mathrm{mol} \mathrm{O}_{2} \mathrm{~g}^{-1}$ biomass $\mathrm{h}^{-1}$ \\
\hline a & 192.00 & 174.00 & $\mu \mathrm{mol}$ photons $\mathrm{m}^{-2} \mathrm{~s}^{-1}$ (PAR) \\
\hline $\mathrm{K}$ & 0.14 & 0.13 & adimensional \\
\hline$\beta$ & 8.50 & 10.00 & $\mathrm{~s}^{-1}$ \\
\hline
\end{tabular}

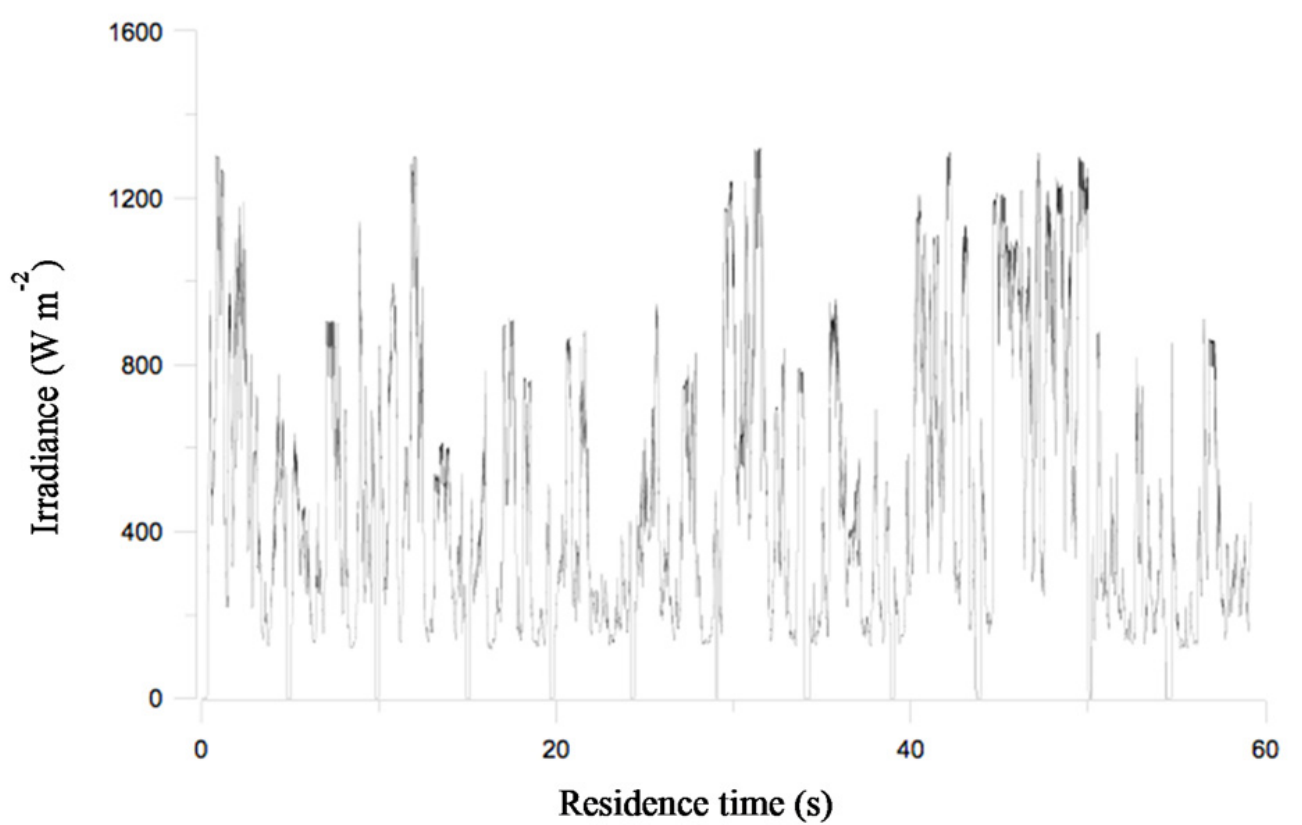

Figure 6. Light regime inside the simulated photobioreactor. The light pattern $I(t)$ that elicits the actual photosynthetic response is a consequence of the movement of the fluid elements inside the irradiance field, both simulated by CFD.

The calculation procedure is straightforward. Equation 1 was integrated with the light pattern $\mathrm{I}(\mathrm{t})$ obtained from the CFD simulation using a $4^{\text {th }}$ order Runge-Kutta method. The integration procedure yields the time evolution of excited photosynthetic centers, $x^{*}(t)$, that allows obtaining the instantaneous photosynthetic response $\mathrm{PO}_{2}(\mathrm{t})$ as photosynthetic oxygen generated (specific oxygen generation as it is expressed per unit biomass) with 
equation (2). This was averaged to obtain the mean photosynthetic response, $\mathrm{PO}_{2}$, during the time of the simulation.

The procedure is summarized in Figure 7. For the case described, the average specific productivity for the time simulated around solar noon, as described in the methods section resulted $\mathrm{PO}_{2}=3.03 \mathrm{~mol} \mathrm{O}_{2} \mathrm{~g}^{-1}$ biomass $\mathrm{h}^{-1}$ which for the biomass concentration in the PBR $\left(C_{b}=2060 \mathrm{~g} \mathrm{~m}^{-3}\right)$ results in a volumetric oxygen productivity of $6.25 \mathrm{~mol} \mathrm{O}_{2} \mathrm{~m}^{-3} \mathrm{~h}^{-1}$. Taking into account the stoichiometry of photosynthesis:

$$
\mathrm{CO}_{2}+\mathrm{H}_{2} \mathrm{O} \rightarrow \mathrm{C}\left(\mathrm{H}_{2} \mathrm{O}\right)+\mathrm{O}_{2}
$$

This means that $1 \mathrm{~mol}$ of $\mathrm{C}$ is fixed for every $1 \mathrm{~mol}$ of $\mathrm{O}_{2}$ released and accepting a $50 \%$ carbon content in the biomass (d.wt) the volumetric biomass productivity of the simulation results in $150 \mathrm{~g}$ biomass $\mathrm{m}^{-3} \mathrm{~h}^{-1}$.

The results of the simulation only take into account the $140 \mathrm{~L}$ of culture in the loop that is where photosynthesis occurs. The PBR contains another $100 \mathrm{~L}$ of culture in dark zones such as the degasser and recirculation pipes. Taking that dark volume into account, the simulation predicts an overall volumetric productivity of $0.108 \mathrm{~g}^{2}$ biomass $\mathrm{m}^{-3} \mathrm{~h}^{-1}$ for the

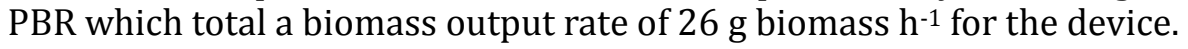

This result agrees with the experimental productivity measured for the PBR described within a $20 \%$ error margin, which demonstrates that the CFD simulation coupled with growth data obtained in laboratory can predict the productivity of the microalgae-based production system, showing that CFD can be used as a design tool for PBRs in order to attain high-performance PBRs and optimize the operating conditions of existing ones.

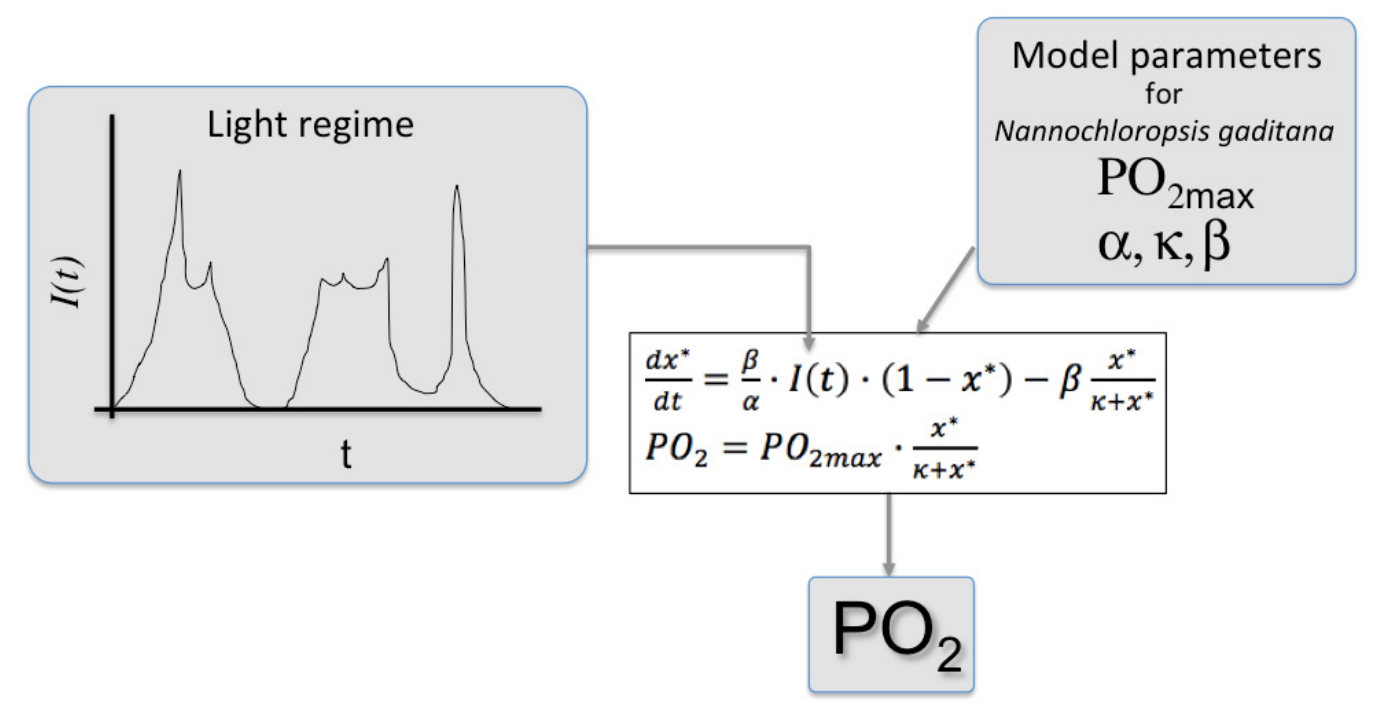

Figure 7. Scheme of the procedure to calculate photosynthetic response from the light pattern obtained from CFD simulation.

\section{FUTURE WORKS} conditions.

Simulations are to be tested in different PBRs and under different operating

\section{Literature cited}

Bitog, J.P., Lee, I.-B., Lee, C.-G., Kim, K.-S., Hwang, H.-S., Hong, S.-W., Seo, I.-H., Kwon, K.-S., and Mostafa, E. (2011). Application of computational fluid dynamics for modeling and designing photobioreactors for microalgae $\begin{array}{lllllll}\text { production: A } & \text { A } & \text { review. } & \text { Comput. } & \text { Electron. } & \text { Agric. } & \text { (2), }\end{array}$ http://dx.doi.org/10.1016/j.compag.2011.01.015.

Brindley, C., Fernández, F.G., and Fernández-Sevilla, J.M. (2011). Analysis of light regime in continuous light 
distributions in photobioreactors. Bioresour. Technol. 102 (3), 3138-3148. PubMed http://dx.doi.org/10.1016/j.biortech.2010.10.088

Chisti, Y. (2007). Biodiesel from microalgae. Biotechnol. Adv. 25 (3), 294-306. PubMed http://dx.doi.org/10.1016/j.biotechadv.2007.02.001

Patankar, S.V. (1980). Numerical Heat Transfer and Fluid Flow (New York: Hemisphere Publishing Corporation, Taylor \& Francis Group).

Rubio, F.C., Camacho, F.G., Sevilla, J.M., Chisti, Y., and Grima, E.M. (2003). A mechanistic model of photosynthesis in microalgae. Biotechnol. Bioeng. 81 (4), 459-473. PubMed http://dx.doi.org/10.1002/bit.10492

Veenman, M.P.B. (2004). Statistical analysis of turbulent pipe flow: a numerical approach. PhD Thesis (Eindhoven, The Netherlands: Eindhoven University of Technology).

Walpot, R.J.E., van der Geld, C.W.M., and Kuerten, J.G.M. (2007). Determination of the coefficients of Langevin models for inhomogeneous turbulent flows by 3D PTV and DNS. Phys. Fluids 19 (4), 045102 http://dx.doi.org/10.1063/1.2717688. 